\title{
CONSIDERACIONES SOCIOLÓGICAS SOBRE EL DESARROLLO DE AMÉRICA LATINA: UNA REVISIÓN DE LOS APORTES DE GERMANI Y MEDINA ECHAVARRÍA*
}

\author{
GUSTAVO RODRÍGUEZ ALBOR**
}

\begin{abstract}
RESUMEN
Desde finales del siglo XVIII, los economistas clásicos y posteriormente muchos de sus seguidores han intentado, explicar el desarrollo económico de las naciones. Sin embargo, la aplicación de sus postulados no han obtenido los mismos resultados frente a los llamados países subdesarrollados como los de América Latina. Conscientes de lo anterior, sociólogos destacados como el italiano Gino Germani y el español José Medina Echavarría en la década 1960, realizan una serie de análisis sobre las posibles causas del subdesarrollo en Latinoamérica, las estructuras sociales que la integran y la forma asincrónica de su transición a la "modernidad". El objetivo de este trabajo es exponer las principales consideraciones de los sociólogos Gino Germani y José Medina Echavarría sobre la sociedad latinoamericana, el importante papel que le brinda a la sociología el estudio de sociedades como la Latinoamericana y valorar la contribución de sus aportes los cuales no son ponderados comúnmente en la literatura económica tradicional. El mensaje central de los autores es que en los países de desarrollo posterior, no se dan algunos de los elementos propios de la modernidad, pues aún subsisten elementos tradicionales de la hacienda feudal, los cuales se dieron de manera totalmente diferente a los procesos de los países industrializados.
\end{abstract}

Palabras clave: Sociología económica, Sociedad, Desarrollo Económico, Modernidad, Sociología Latinoamericana.

\footnotetext{
* El presente ensayo fue sustentado y debatido en el marco del Doctorado en Ciencias Sociales, válido como requisito para aprobar el Módulo Pensamiento Sociológico Latinoamericano, en Noviembre de 2009. La versión original se actualizó y amplió entre 2010 y 2011. El autor es el único responsable de las ideas que aquí se presentan.

** Economista y candidato a Doctor en Ciencias Sociales de la Universidad del Norte (Colombia). Investigador y Docente Catedrático de la Escuela latinoamericana de Cooperación Internacional y Desarrollo de la Universidad de San Buenaventura de Cartagena (Colombia) en convenio con la Universitá di Pavia (Italia). Investigador y Docente Catedrático de la Universidad del Norte (Colombia). Correo Electrónico: gustavo829@gmail.com.
} 


\begin{abstract}
Since the end of the 18th century, classical economists, and subsequently many of his followers have attempted to, explain the economic development of Nations. However, the implementation of its postulates not obtained the same results with the so-called underdeveloped countries such as Latin America. Aware of this, sociologists highlights such as the Italian Gino Germani and Spanish José Medina Echavarría during the 1960, carried out a series of analyses on the causes of underdevelopment in Latin America, the social structures that make up and the asynchronous form of the transition to "modernity". This work aims to outline the main considerations of the Gino Germani and José Medina Echavarría sociologists on the Latin American society, the important role that affords the sociology study of societies such as the Latin American and assessing the contribution of their contributions which are not commonly weighted in traditional economic literature. The central message of the authors is that in the countries of further development, fail some of the elements of modernity, because traditional elements of the feudal estate, which were totally differently to the processes of the industrialized countries are still.
\end{abstract}

Key words: Economic Sociology, Society, Economic Development, Modernity, Latin American Sociology.

Classification Journal Economic Literature (JEL): A14, O12, O15

\title{
INTRODUCCIÓN
}

Desde finales del siglo XVIII, los economistas principalmente los clásicos y neoclásicos, han intentado explicar el crecimiento y el desarrollo económico de los países. A pesar de ello, es con el fin de la segunda guerra mundial, que se inicia el auge de las teorías del desarrollo, básicamente bajo la concepción neoclásica, las cuales se construyeron a partir de las dinámicas propias de los países desarrollados en una época en la que el capitalismo se encontraba en plena "edad de oro" y en donde la revolución industrial impulsaba enormes cambios sociales, para ser replicadas posteriormente en las llamadas "economías subdesarrolladas". Sesenta años después de la "maratón" de modelos de desarrollo implementados en países como los de América Latina, la evidencia empírica demuestra que han fracasado en su afán de que sus economías alcancen el desarrollo (1).

Testigos del auge de las explicaciones del desarrollo a mediados de los cincuenta, sociólogos destacados como el italiano Gino Germani y 
el español José Medina Echavarría en la década de 1960, analizan por fuera del paradigma neoclásico, las posibles causas del subdesarrollo en Latinoamérica, a partir de aspectos tales como las estructuras sociales, la dependencia económica, la secularización, la estructura hacendataria y las burguesías, entre otros elementos, que consideraron no permiten que los mismos esquemas de desarrollo que triunfan en Europa y Estados Unidos se consoliden, por lo menos totalmente, en Latinoamérica.

El presente ensayo tiene como objetivo realizar una aproximación de la perspectiva sociológica más relevante relacionada con la estructura social y el desarrollo en América Latina propuesta por estos dos importantes autores. Para ello, inicialmente se realiza una breve descripción del debate acerca de la explicación sobre desarrollo económico y el surgimiento de la sociología económica como un nuevo campo de estudio. Luego, se exponen las influencias de pensamiento que tuvieron tanto Gino Germani, como José Medina Echavarría para crear sus reflexiones y consideraciones acerca del desarrollo económico latinoamericano, desde la perspectiva sociológica. Finalmente, se hace una serie de valoraciones de los aportes de estos sociólogos.

Este ensayo igualmente pretende que se valore el análisis económico que puedan brindar otras disciplinas más allá del paradigma neoclásico, de tal manera que se incorpore en la investigación social la metodología científica y el carácter crítico que ofrecen estos campos de estudios donde los factores sociológicos y otros relacionados con la naturaleza humana, hacen parte de la realidad explicada.

\section{LA EXPLICACIÓN DEL DESARROLLO: ¿ECONÓMICA O SOCIOLÓGICA?}

Terminada la segunda guerra mundial, la necesidad de buscar explicaciones al desarrollo de los países fue una tarea encomendada exclusivamente a los economistas, y aunque desde 1776, Adam Smith y otros economistas clásicos se preocuparon por las razones del crecimiento de las naciones, sólo hasta la década de los años cincuenta se comienzan a establecer como una tarea científica encontrar los factores que conducen al desarrollo económico. Desde entonces la teoría neoclásica ha tenido gran aceptación, donde el paradigma central se basa en que los seres humanos son individuos racionales que maximizan la utilidad y minimizan sus costos, y que tal comportamiento es en gran medida invariable a través de diferentes sociedades humanas. 
En este sentido, la economía neoclásica moderna, tiende a restarle importancia al papel que juega los factores culturales, sociológicos y antropológicos en el desarrollo económico. Los modelos de crecimiento económico como los de Lewis, Solow, Arrow, Harrod - Domar y Uzawa; entre muchos otros, sólo tenían presente capital y mano de obra y más recientemente, como los de Barro y Lucas, han "endogenizado" a estos la tecnología y el conocimiento ${ }^{1}$. Desde la perspectiva de estos economistas, la cultura como máximo constituye una especie de factor residual al que se recurre cuando fallan otras explicaciones (Fukuyama, 2001, p. 3130). Así, el papel de los factores sociológicos y culturales en el desarrollo económico de las sociedades ha sido estudiado más por los sociólogos que por los propios economistas.

Bajo esta perspectiva, la explicación sociológica de los eventos económicos tiene sus antecedentes en los representantes de la sociología clásica como Weber, Durkheim y Simmel, los cuales consideraron fundamental desarrollar este tipo de análisis de los fenómenos económicos para poder comprender la sociedad. Para Weber (3), por ejemplo, el protestantismo fue el elemento vital para el proceso de industrialización europeo y forjar el desarrollo capitalista. Asimismo, Simmel consideró que la economía es intercambio, es una forma elevada y por antonomasia del intercambio social. Considera que el intercambio es primero social, y luego económico; y que si se valoran correctamente estas formas de intercambio social se puede llegar a comprender los resortes psíquicos o motivacionales del intercambio económico. Emile Durkheim (4), por su parte criticó la forma de análisis económico. Sostuvo este sociólogo, que los mercados descritos por los economistas presuponen la existencia de normas generalizadas no teniendo en cuenta conductas particulares de los individuos, en contraste con la sociología que busca analizar los fenómenos a un nivel más profundo de causalidad. Igualmente, Durkheim sugirió que en una sociedad capitalista, con una compleja división del trabajo, la regulación económica sería necesaria para mantener el orden. Al respecto, Karl Mannheim (5) argumentó que el paso a un mercado autorregulado implica una transformación del mecanismo de regulación y control social sobre el principio mismo de organización de la propia sociedad.

A pesar de tales consideraciones, sólo hasta mediados de los años ochenta surge un grupo de sociólogos e intelectuales de las ciencias sociales con la inquietud de debatir y criticar algunos principios del paradigma

\footnotetext{
${ }^{1}$ Meier, G. (2), ofrece una descripción sintética de la evolución de los modelos neoclásicos en su artículo "La Vieja Generación de los economistas del Desarrollo y la Nueva". En: Fronteras de la Economía del Desarrollo. Meier, G. \& Stiglitz, J. (Editores).
} 
económico predominante, particularmente lo relacionado con el concepto de racionalidad instrumental y el individualismo metodológico en los que la teoría neoclásica moderna fundamenta su análisis de la acción económica, lo que abrió paso a un nuevo campo de estudio conocido como la sociología económica. Para Pozas (6) los postulados teóricos básicos de la sociología económica son: primero, la acción económica es una forma de acción social, segundo, la acción económica está socialmente situada o imbricada en una estructura y, tercero, las instituciones sociales son construcciones sociales. Así la sociología económica surge como una ciencia encaminada a proporcionar una comprensión institucional con experiencia y riqueza cultural de la vida económica, buscando explorar la relación entre la economía y la sociedad durante un período histórico de transformación intensa (7).

Más recientemente, la sociología económica se ha caracterizado por un crecimiento muy dinámico de los tópicos de estudio que incluyen la riqueza, el emprendimiento, los mercados, el comportamiento económico y el desarrollo. Para Nee y Swedberg (8), las ideas iniciales han sido trabajadas y se han desarrollado en nuevas direcciones. También se han agregado nuevos temas para su programa, y se han logrado avances interesantes en el análisis de algunos de los temas que se debatieron durante su fase inicial en la década de 1980 (9).

Estos nuevos campos de estudios económicos basados en el comportamiento social del hombre han ganado cada vez más una mayor aceptación de la comunidad científica. Así por ejemplo, la economía institucional ha tomado varios elementos de este campo de estudio para buscar explicaciones sobre el desarrollo, a partir de factores como la cultura, las conductas y hábitos de los individuos, de los cuales han surgido nuevas aplicaciones en el campo de la economía como son los costos de transacción y la teoría de cumplimiento de los contratos, entre otras.

\section{CONSIDERACIONES SOCIOLÓGICAS SOBRE EL DESARROLLO ECONÓMICO DE AMÉRICA LATINA}

Como se describió, el auge de los estudios a partir de la sociología económica ha tenido una tendencia creciente en el ámbito científico social y nada parece indicar que esto va a disminuir. En el caso de América Latina, la creciente popularidad de la sociología económica se explica además por el agotamiento de los estudios empíricos realizados desde una perspectiva estructuralista de tradición marxista, y la pérdida de 
presencia real de los actores colectivos tradicionales como fueron los sindicatos, el Estado, los movimientos sociales y los partidos, entre otros (6). En términos generales, los estudios que buscan darle explicación a los diferentes niveles de desarrollo económicos de los países se han proliferado, en especial en aquellos donde los elementos culturales parecen ser relevantes.

Por ello, es importante reconocer los primeros intentos de encontrar una explicación del desarrollo en América Latina por fuera de la teoría neoclásica, muchos años antes del surgimiento y reconocimiento de la explicación sociológica como argumento válido para entender los fenómenos económicos. En este sentido, resulta interesante destacar los aportes del sociólogo español José Medina Echavarría y del italiano Gino Germani, a la evolución económica de América Latina.

En el caso José Medina Echavarría, fue reconocido por concebir a la sociología, por una parte, como una ciencia reveladora de la condición convencional de toda construcción social y, por otra, porque observó desde un principio el posible carácter instrumental y crítico de su papel y función en la sociedad (10). Medina fue discípulo de José Ortega y Gasset y su perspectiva sociológica se inspiró en la sociología alemana y, en este sentido, la obra de Max Weber constituye su principal centro de atención e interés. Sus obras se surten de pensadores como Simmel, Mannheim, Marx, Veblen y Tönnies, entre otros, todos ellos considerados precursores de la actual sociología económica.

Por su parte, Gino Germani intenta en sus obras sintetizar las sociedades contemporáneas al igual que lo hicieron Ferdinand Tönnies y Max Weber. Construye su análisis estructuralista de la sociedad a partir de Talcott Parsons y Robert K. Merton. Precisamente, la interpretación de Parsons es fundamental para tomar los elementos de la acción e introducirlos a la teoría de la acción social la cual se convierte, según el autor, en una vía aplicable a la planificación integradora que el desajuste de la sociedad global requería para su continuidad. Afirmaba Germani que los procesos en América Latina se hacen diferentes a los europeos aunque indicó que todavía no tenía una base empírica para confirma su tesis.

La base del análisis de su principal obra sobre el desarrollo latinoamericano fue la "Política y Sociedad en una época en Transición: De la sociedad tradicional a la sociedad de masas" es Kart Mannhein al utilizar sus categorías de los ensayos de sociología y psicología social. En su trabajo también se traslucen las formas de solidaridad de Durkheim, la racionalidad y la 
intencionalidad histórica de la acción social de Weber, la interdependencia y funcionalidad de partes generalizadas de Parsons (11).

\section{Postulados centrales de Medina Echavarría: La Estructura Social y las Burguesías}

En su principal obra sobre el desarrollo latinoamericano "Consideraciones Sociológicas sobre el Desarrollo Económico en América Latina" Medina se propone como objetivo considerar el desarrollo de América Latina en sus aspectos sociales. La postura del autor es la de esforzarse por poner al menos un poco de orden en la confusión de las mezcladas significaciones que existen sobre el tópico del desarrollo. Considera que el desarrollo económico supone un proceso de continuas inversiones, pero se le brinda mayor importancia a las de carácter económico, que a las inversiones humanas (Ej. Salud y educación). Estas se han convertido en categorías o indicadores de planeación (Tecnificación del problema), sin embargo, para abordar el desarrollo económico no puede basarse sólo en indicadores socioeconómicos, que a la postre no lo solucionan. El lema del desarrollo económico y social equilibrado es una exigencia y no parecen integrarse en la realidad. El desarrollo para Medina, posee un elemento utópico. Las exigencias de la práctica (o del conocimiento) radican en que las orientaciones de política social presentan deficiencias. Considera que es una tarea la integración conjunta de la planeación social y económica.

Medina plantea en su libro una serie de consideraciones o planteamientos acerca del problema del desarrollo en las sociedades latinoamericanas mediante un diagnóstico sociológico. Parte de que el diagnóstico como interpretación de una situación, sólo se logra si se tiene una idea de su estructura y de las tendencias dinámicas que en ella se manifiestan. Las posibilidades de realización dependen de las condiciones externas de una coyuntura (12)

El principal cuestionamiento de Medina Echavarría es ¿Cómo enfrentarse al problema del desarrollo? Al respecto, considera dos elementos; el primero es el problema histórico en el análisis de la estructura social Latinoamericana y, el segundo, es la aparición de las burguesías, las cuales aunque podrían considerarse legítimas por su tradición en Latinoamérica, no han resultado ser eficaces.

Para Medina, la revolución industrial no se consolidó en América Latina. Mientras que en las sociedades industriales se encuentra en la segunda fase, América Latina pasa por los esfuerzos de la primera sin sustraerse de los 
efectos favorables o adversos de la segunda. Afirma que la estructura de la sociedad latinoamericana estaría constituida por la coexistencia de dos sociedades distintas a lo que llama "dualismo estructural de la sociedad latinoamericana", dado que esta puede ser moderna y tradicional, progresiva y arcaica, protectora y opresora, autoritaria y paternal, fiel y resentida. Considera que esta concepción sociológica es ideal, pues los países avanzados en sus aspectos sociales no han tenido rupturas bruscas, sino diferenciaciones continuas.

Otro aspecto que destaca el autor español es el escaso o poco papel que han jugado los intelectuales y las universidades en el desarrollo de América Latina. Con respecto al discreto desempeño de las universidades a lo que denomina gravísimo tema, Medina (12) comenta "el crecimiento económico es deficiente porque no cuenta con el necesario apoyo técnico e intelectual de la universidad, como al contrario la universidad es deficiente porque el país que la sostiene no cuenta con la suficiente riqueza" (12). Hace un llamado a los responsables de que a pesar de que para 1550 ya existían universidades en Latinoamérica estos centros permanecieron intactos incluso hasta después de la independencia y que en la actualidad su evolución es lenta. Con respecto a este mismo tema termina insistiendo en que "hagamos todo lo posible por elevar el nivel de la universidad, para que ésta sea un instrumento eficaz del crecimiento económico y social, pero muy en particular no deje de ser el pouvoir spiritual que todavía puede encarnar" (12).

Medina también aborda el tema de la urbanización acelerada y la explosión demográfica. Considera que el rápido crecimiento de la población urbana en América Latina, no se debe tanto a su crecimiento natural como a la emigración de las masas campesinas (desarraigados) que son casi imposibles de absorber. Estas son expelidas más por las deficiencias de las estructuras agrarias que por la atracción al sistema industrial incipiente de las ciudades. En consecuencia, sólo se ha transferido el problema del campo a la ciudad.

Otro aspecto que es posible destacar de las consideraciones de Medina es la persistencia de la imagen paternalista. El patrono demagogo reemplaza al hacendado paternalista. Las masas suelen ser arrastradas por líderes carismáticos y vulnerables a influencias extremistas tanto de derecha como de izquierda. 


\section{Las estructuras sociales y de poder tradicional}

Para Medina Echavarría una de las instituciones que reviste especial atención, con el firme objetivo de poder entender el proceso de transformación que experimenta la sociedad latinoamericana desde las primeras décadas del siglo XX, lo constituye la hacienda. El sentido sociológico que tuvo esta institución en América se puede apreciar mediante la propia caracterización que realiza dicho autor:

"Toda estructura social suele ofrecer en sus partes más diversas, en sus lugares más inesperados, la huella y el influjo de un determinado prototipo. Las actuales sociedades industriales reciben la impronta del establecimiento fabril en relaciones y modos de vida muy alejados y sin conexión aparente con ese centro de producción. La estructura social de América Latina mostró por largo tiempo en todos sus entresijos la capacidad modeladora de una institución fundamental: La hacienda. Toda la historia económica, social y política de América Latina es en buena parte la historia de la consolidación y transformaciones de esa unidad económico-social. Y el relato del ocaso de la estructura tradicional se confunde por consiguiente con el lento declinar de esa vieja organización. Ocaso y no extinción, desde luego, pues todavía persisten tanto su presencia como sus influjos" (12).

Para Medina la hacienda hizo a América Latina predominantemente agraria (hasta hoy) y en ella es posible distinguir un conjunto de rasgos tales como "a) el haber sido célula de poder político-militar al lado del económico; b) el haber constituido el núcleo de una dilatada estructura familística; c) el haber constituido el modelo circunstancial de la autoridad, d) el haber sido la creadora de un tipo humano de un carácter singular" (12), es decir el padre hacendado, tomando la hacienda un papel no solamente económico, sino también de protectora. Estos rasgos justifican en buena parte la razón de la fuerte y prolongada permanencia de una institución que, en términos económicos, presentaba muchas insuficiencias y limitaciones. A juicio de Medina:

"La hacienda desde su cristalización originaria es algo más que una unidad de producción económica. Es un instrumento de instalación de un orden en el dilatado espacio vacío del agro, y por tanto significa de hecho un núcleo de poder político, tolerado o utilizado según circunstancias por las autoridades estatales, y al que se otorga a veces o toma por sí mismo una significación militar" (12). 
En definitiva, la hacienda poseía una gran centralidad al ser el espacio en torno a la que se concentraban mayoritariamente las relaciones económicas y sociales, configurando la principal fuente de poder del orden oligárquico el cual se traducía en el control absoluto del aparato estatal por parte de las élites.

\section{La aparición de las burguesías}

El carácter elitista y excluyente del sistema político empieza a ser modificado con la emergencia de los sectores medios y obreros urbanos a comienzos del siglo XX. Las élites cosmopolitas (educados en Europa), son reemplazados poco a poco por nuevos grupos dirigentes (la nueva clase media) con cierto grado de educación, prósperos y auténticos quienes se rebelan ante la realidad interior. A partir de ese momento, el conflicto de poderes desplazado por las presiones de mayor integración por parte de esos nuevos sectores. Este último hecho, junto a otros factores como el incremento de la urbanización y la mayor complejidad que asume la estructura social, provocan el agotamiento de las instituciones políticas y sociales prevalecientes.

A pesar de esas transformaciones, gran parte de las haciendas logra mantener su predominio. Incluso, pese a que desde fines del siglo XIX en una gran cantidad de estas unidades se dan ciertos niveles de modernización, en el sentido de Medina, no logran transformarse verdaderamente en empresas.

Para Medina, se conforma una nueva estructura de poder con el tránsito de un sistema histórico (el de Hacienda) a otro absorbente de futuro (el sistema industrial). Considera que el viejo sistema de hacienda funcionó con mayor eficacia y legitimidad. El ocaso acompañado del sistema bipartidista tradicional y de hacienda es resultado de la transformación de la estructura social (la aparición de la clase media y la descomposición ideológica). Ese vacío político y de legitimidad dejado por la oligarquía secular, se mantiene en la estructura de poder de los partidos políticos. Puntualmente Medina (12) anota:

"El hueco de la estructura de poder que mantiene todavía la inadecuada transformación de los partidos políticos históricos que forjó en su momento - y con acierto- el sistema de hacienda es un vacío gravísimo porque deja en el aire las raíces de la legitimidad. Un régimen para ser creador - no para mantenerse más o menos tiempo - ha de ser, se ha dicho repetidamente (desde Aristóteles a Max Weber y por todos los 'maquiavelistas' sin 
excepción) a la par legítimo y eficaz. Pero si mucho se aprieta es más importante lo primero que lo segundo. No es imposible que las viejas clases - las oligarquías de otrora- sean capaces de ganar una nueva legalidad si se esfuerzan por modificar a la altura de los tiempos su formula política" (12).

En este proceso esa clase media emergente parece ser la más apta para ver por la enorme masa de desarraigados e impulsar el desarrollo económico, pues las clases altas (burguesas tradicionales) no están interesadas en ello y la clases bajas no poseen ningún tipo de poder. Sin embargo, muchos de los integrantes de esa nueva clase han terminado conformando e integrando a esa clase burguesa (tendencia al arribismo). Desde otra perspectiva, es importante destacar que la crisis de la burguesía más tradicional suscitada en esta etapa tampoco logra alterar la persistencia de las haciendas.

\title{
El enfoque de Germani: Modernización, Burguesía y la Acción Social
}

La tesis central argumentada por Gino Germani en su obra "Política y Sociedad en una época en Transición: De la sociedad tradicional a la sociedad de masas" sobre el desarrollo económico y de los cambios sociales es que los procesos de modernización en América Latina, se hacen de forma diferentes a los del europeo, generando por lo tanto efectos diferenciales, debido a que presentan fenómenos sociales diferentes de las llamadas sociedades capitalistas occidentales. En este sentido Germani afirma:

\begin{abstract}
"El surgimiento y desarrollo de la sociedad industrial requiere la 'movilización' y de hecho se acompaña de ella, de la totalidad de todos los habitantes de un país. En el modelo occidental tal 'movilización' ha consistido también en la movilización política. Más la transición presenta en los países de desarrollo posterior -como en el caso de América Latinarasgos diferenciales de esencial importancia" (13).
\end{abstract}

Amplía esta idea el autor clasificando en 3 grupos esos fenómenos sociales en los que se dan esas diferencias, así: (a) La estructura social, la cultura y los tipos de personalidad de los países (b) La secuencia y rapidez de los cambios y (c) La época histórica. Considera el análisis de la transición un proceso de secularización (en el conocimiento, la técnica y la economía). Por ello, el cambio es asincrónico, pues este no se da de forma igual por regiones, clases y personas. Para Germani, la asincronidad fue el rasgo esencial de los procesos de cambio o modernización en América Latina. 
Uno de los aspectos más destacados de la diferenciación es la burguesía latinoamericana que no dejó que se realizara un proceso de modernización totalmente liberal e integrador. De acuerdo a Germani, la burguesía ha tomado un papel en la sociedad, es una clase social feudal que tuvo que moverse dinámicamente. Introduce el principio dominante de la acción social: dominación en el individuo y no en la sociedad. En su análisis sociológico, el cambio es el impulso que le da la burguesía al nuevo mundo, ella implica la desintegración de las unidades locales e ingresa una categoría importante "el individuo" (libertad, igualdad) postula la acción social utilizándolo para América Latina.

Considera Germani que debe hacerse un juicio para el análisis de funcionalidad y disfuncionalidad. Germani intenta sintetizar las sociedades contemporáneas y al igual que lo realiza Tönnies, Weber, Marx, entre otros, tomando todas las teorías de la modernización y haciendo énfasis en los elementos que conforman esa modernización.

Estaba seguro que los movimientos poblacionales del campo a la ciudad desarticulaban el espacio social tradicional que intentaba sostenerse marginando a los nuevos actores sociales de la sociedad moderna. Los trabajadores rurales y urbanos con oficios tradicionales se transforman en "obreros", es decir, Germani identifica dos tipos de masas populares: la élite sindical y la campesina con bajo nivel educativo, a las que considera son masas sociales impacientes. De la solución de este conflicto derivaba la estabilidad del Estado-nación vigente. Esta es precisamente la unidad de análisis de su estudio el "Estado-Nación".

Para Germani (13), las élites para mantener su posición manipulan las masas y para ello "emplean las ideologías, como mero instrumento de dominación. Hay una separación entre los propósitos de las élites y los de las masas". Además de ello, en la cultura de sociedades como las Latinoamericanas, Germani encuentra que existe como elemento esencial un alto grado de autoritarismo. El actor de la modernidad son las élites, las cuales tienden a cerrarse sobre sí mismas y a adoptar una cuestión desarrollista, pero en su interior hay una ideología tradicionalista (valores, costumbres). Puntualmente afirma:

Pero en la cultura de las sociedades tradicionales también se halla como elemento esencial del autoritarismo; por otra parte, los valores relativos, en tanto propios de la cultura particular (la occidental) considerada en parte o totalmente, extraña a la cultura nacional, son por ese motivo rechazados. Un proceso correspondiente se da en la masa; subsisten en ella los valores 
tradicionales (entre ellos el autoritarismo), que por efecto de la masificación y la acción politica de las élites llegan a fusionarse con las ideologías autoritarias. Así autoritarismo tradicional y autoritarismo ideológico se refuerzan el uno al otro en las masas populares" (13)

En general se afirmaba en el siglo XIX, que las clases bajas se inclinan por la izquierda mientras que las altas se inclinan por la derecha. Sin embargo, el siglo XX generó un clima político diferente por el fascismo y el estalinismo, las cuales fueron respaldadas por el pueblo. En este sentido, la izquierda también toma elementos de derecha.

Para construir el anterior análisis, Germani parte de 3 dimensiones: Cultura, Sociedad y Motivacional. Existen además estructuras parciales, instituciones, grupos sociales y categoría nominales (eventualmente grupos sociales). Tres aspectos fundamentales cobran un papel preponderante en el proceso del cambio social: El tipo de acción y la preponderancia en la modernidad de las acciones electivas; la institucionalización del cambio; y la diferenciación y la especialización creciente de las instituciones. Argumenta Germani que existe 3 niveles de interacción: Simple interacción de las partes, ajuste recíproco de las partes y adecuación de las partes.

Para la acción social toma los siguientes elementos (1.) Actor (Individuo, Grupo), (2.) La Situación (a. Fines b. Medios c. Condiciones) y el (3.) Marco normativo (a. Normas y pautas, b. Valores c. Conocimientos). Particularmente, consideraba que existen dos formas fundamentales de marco normativo: la acción prescriptiva y la acción electiva. "La acción prescriptiva se caracteriza por desenvolverse en un marco normativo sumamente rígido [....] mientras que en la acción electiva "el marco normativo no sólo es menos rígido sino que actúa de manera diferente" (13). En el caso de no existir o ser insuficiente el marco normativo Germani afirma que el concepto que aplica es el de anomia. En este sentido, Germani considera que la acción social en América Latina se dio de manera anomica y al respecto comenta:

"El problema de la anomia se vincula de manera estrecha a la acción social y en particular con el tema de la racionalidad de la acción, y es por eso que ha sido recordado expresamente aquí. Además, en una consideración más detenida habría que examinar las diferentes posibilidades que se dan una situación de anomia desde el doble punto de vista del observador y del actor, tanto cuando se trate de anomia sociocultural como de anomia psicológica" (13). 
En síntesis, para Germani en los países de desarrollo posterior, no se dan algunos elementos propios de la modernidad, sino que permanecen rasgos de particularismo feudal y siguen hasta hoy en día (ejemplo, el caudillismo, guerras civiles, anarquía). En Europa y otros países avanzados no es así, este es el mayor aporte de Germani a la explicación de la realidad social y económica de América Latina.

\section{Otras consideraciones relevantes sobre América Latina}

Junto a los aportes brindados por Germani y Medina Echavarría, es posible destacar contribuciones relevantes a una visión sociológica del desarrollo latinoamericano. Dentro de estos exponentes se pueden destacar a Sergio Bagú, Gilberto Freyre, George Foster, Fernando Ortiz, José Luis Romero, Glen C. Dealy, Howard J. Wiarda, Frederick Turner y Lawrence Harrison, entre otros.

Así por ejemplo, para Bagú (14), es necesario comprender la situación de la época colonial de América Latina, pues en ella hay una estructura histórico-social, como es por ejemplo, la concepción de castas y clases, la cual se refleja hoy. Asimismo, Freyre (15), explica a partir del caso de Brasil, que la forma social de hoy es producto de la mezcla cultural generada desde el mismo período de la colonia. Por otro lado, Ortiz (16), realiza un análisis comparativo de dos polos de la sociedad latinoamericana a través de dos elementos constitutivos: el cultivo artesanal de tabaco y la agroindustria capitalista del azúcar que vivió Cuba. Introduce el término "transculturación", el cual es hoy muy común para explicar algunos elementos negativos de los procesos de globalización. Foster (17), por su parte considera los países que integran esta región poseen una misma base cultural derivada de sus antiguos dominadores, la que denominan "cultura Iberocatólica", lo que ha influido notablemente en el desarrollo económico de los países de Latinoamerica.

Diversos investigadores también creen firmemente que algunas características histórico-culturales y sociales pueden ser una de la fuente de los "males" de América Latina (18-22). Tal como lo demuestra North (23), Wiarda (22) también considera que la cultura que caracteriza a América Latina difiere ampliamente dela que poseen las Naciones Norteamericanas. En su opinión, "religión, cultura, valores, idioma, derecho e historia son también de una importancia crucial en la comprensión del marco institucional a través del cual el desarrollo es anticuado". Para este autor, a nivel de sus interacciones sociales, Latinoamérica aún conserva 
una estructura jerárquica, autoritaria, católica, elitista, corporativista y paternalista de la colonia.

En esta misma perspectiva, Dealy $(1977,1992)$, caracteriza la cultura de América Latina como de "caudillaje". En sus palabras, "el Hombre de caudillaje activamente y racionalmente utiliza el ocio para el adelanto de su objetivo de poder público". Medina Echavarría (12) también destacó al caudillismo como una característica en la cultura del hombre latinoamericano. El recientemente laureado escritor peruano Vargas Llosa (24), afirma que las reformas que se requieren en la economía de América Latina, el sistema de educación y la administración de justicia son prácticamente imposibles de realizar. Y de ser posibles, están condenadas a ser efímeras, "a menos que sea precedido o acompañado por una reforma de nuestras costumbres y ideas, del conjunto complejo sistema de hábitos, conocimientos, imágenes y formas que se entiende por "cultura". Consideraciones como las anteriores ven como un gran obstáculo para el desarrollo económico la cultura latinoamericana.

\section{VALORACIÓN DE LOS APORTES}

Tanto Gino Germani como José Medina Echavarría realizan una serie de importantes aportes, en el ámbito de la sociología latinoamericana, para el entendimiento del problema de las estructuras sociales latinoamericanas, el papel de la burguesía y en general de su desarrollo o crecimiento económico desde la perspectiva sociológica.

Entre esos aportes cabe destacar la importancia de la obra de José Medina Echavarría "Consideraciones sociológicas sobre el desarrollo económico", ya que permite identificar aquellos aspectos que subsisten y coexisten por varias décadas del siglo XX en buena parte de los países de América Latina. Tales aspectos influyeron para que la modernización estuviese condicionada por paulatinos procesos de tránsito y de transformación de ciertas estructuras e instituciones económicas y sociales.

Por su parte Germani, basa su análisis estructuralista de la sociedad a partir de Mentor y Parson. En su obra "Política y Sociedad en una época en Transición: De la sociedad tradicional a la sociedad de masas" la búsqueda de sentido en las acciones de las masas populares, la comparación entre los totalitarismos, el estudio del proceso de secularización como transición de una situación "tradicional" a otra de "modernidad", la urbanización y el análisis de la falta de sincronía en la integración, son reflexiones que brindan un nuevo enfoque sobre procesos sociales en América Latina. 
Asímismo, al revisar el enfoque teórico que sustenta la ideología sociológica del análisis de Medina se basa principalmente en José Ortega y Gasset y Max Weber; por su parte Gino Germani se fundamenta principalmente en el análisis de las estructuras de Mentor y Parson. Por otro lado, mientras Medina Echavarría toma algunos de elementos del socialismo soviético para realizar comparaciones como el modelo liberal, Germani toma una postura alejada tanto de los pensamientos de izquierda, como de derecha, en parte por que sufrió las consecuencias de la persecución del fascismo de Mussolini y el peronismo en Argentina.

Finalmente, en cuanto a las diferencias fundamentales relacionadas con las consideraciones de estos autores, radican principalmente en elámbito que le dan a los elementos que analizan. Así por ejemplo, mientras Medina Echavarría le brinda un papel fundamental a los intelectuales y especialmente a la universidad en los procesos de desarrollo de los países, Germani no se preocupa mucho por este tema. Con respecto a las estructuras sociales y las masas, Medina Echavarría considera que el sector de las clases medias pueda construir una estructura, preocupándose en que medida serán aptas para impulsar el desarrollo económico, por el contrario Germani no le da tanto peso a esa clase media emergente. Germani enfoca más su análisis a darle respuesta al populismo.

Además de lo anterior, es importante destacar la extraordinaria contribución de ambos autores con el esquema metodológico para el estudio científico de la situación de Latinoamérica, convirtiéndose en dos pensadores de la realidad social de esta región, desde un punto de vista diferente al que se acostumbra a emplear tradicionalmente para explicar este tipo de fenómenos.

\section{CONSIDERACIONES FINALES}

En el marco de la sociología latinoamericana resulta de vital trascendencia retomar las diferentes contribuciones de las obras de Gino Germani y José Medina Echavarría sobre los diferentes fenómenos relacionados con las estructuras sociales y la situación económica de esta región.

Ambos sociólogos sin apartarse de la rigurosidad del análisis científico, ponen de manifiesto sus respectivas tesis sobre las diferentes dinámicas a que se ha enfrentado la sociedad de América Latina al incorporarse de manera abrupta a los procesos de modernización, que en Europa y habían iniciado tres siglos atrás. Este proceso acelerado desencadenó un efecto 
diferencial que en la actualidad siguen inmerso en las diferentes naciones latinoamericanas.

Las obras de Gino Germani, José Medina Echavarría y otros sociólogos económicos se distinguen por el alcance de su aplicación interpretativa de una realidad única como es la posee la sociedad latinoamericana, contribuciones que son poco valoradas por la sociología contemporánea latinoamericana y aún menos por los teóricos del desarrollo.

Resulta por lo tanto importante, incorporar en las explicaciones e investigaciones sociales actuales la metodología científica y el carácter crítico de estos autores, pues las estructuras tradicionales que revelan en sus análisis provenientes desde el sistema feudal, aún hoy se encuentran en las diferentes esferas de la sociedad, lo que justifica la vigencia de sus tesis y la aplicabilidad de las mismas.

\section{REFERENCIAS BIBLIOGRÁFICAS}

1. Easterly, William. En busca del crecimiento: Andanzas y tribulaciones de los economistas del desarrollo. Antoni Bosch editor. 2003.

2. Meier, G. La Vieja Generación de los economistas del Desarrollo y la Nueva. En: Fronteras de la Economía del Desarrollo. Meier, G. \& Stiglitz, J. (Editores) Banco Mundial y Alfaomega. P. 2 y 3. 2001.

3. Weber, Max. La ética protestante y el espíritu del capitalismo. Edición en español del Fondo de Cultura Económica 2003. México. P. 277-278. 1930.

4. Durkheim, Emile. La División del Trabajo en la Sociedad. Trans. Lewis A. Coser. New York: Free Press, 1997, pgs. Nueva York: Free Press, 1997.

5. Mannheim, Karl. Freedom, Power, and Democratic Planning. New York: Oxford University Press. 1950.

6. Pozas, María de los Ángeles. Aportes y Limitaciones de la Sociología Económica. En: Cuaderno de Ciencias Sociales 134: La Sociología Económica: Una Lectura desde América Latina. Pozas, M., Mora, M. y Pérez, J. (autores). Facultad Latinoamericana de Ciencias Sociales (FLACSO). Costa Rica. P. 9 - 36. 2005. 
7. Guillén, Mauro F. Comparative Economic Sociology: Blending Social Stratification, Organizational Theory, and the Sociology of Development. Prepared for Presentation at the Latin American Studies Association Annual Meeting Miami, 2000. p. 1 - 14. 2000.

8. Nee, Victor y SWEDBERG, Richard. Economic Sociology and New Institutional Economics. En: Handbook of New Institutional Economics. C. Ménard and M.M. Shirley (eds). p.789 -818. 2005 Springer. 2005.

9. Swedberg, Richard. On the Present State of Economic Sociology (1990s). Economic Sociology. European Electronic Newsletter. Vol. 5, No. 2 (January 2004). P. 2 - 17. 2004.

10. Rodriguez, M. José Medina Echavarría (1903-1977): La sociología como ciencia social concreta. Rev. Política y Sociedad 2004, Vol. 41 Núm. 2: 11-29. Facultad de Ciencias Políticas y Sociología. Universidad Complutense de Madrid. P. 11. 2004.

11. Tavella, A. M. Gino Germani: El Oficio Del Sociólogo en la Argentina. Instituto de Investigaciones Gino Germani. Universidad de Buenos Aires. P. 13. En: http://www.iigg.fsoc.uba.ar/germani.htm. 2007.

12. Medina Echavarría, José. Consideraciones Sociológicas sobre el Desarrollo Económico en América Latina. Editorial EDUCA. Edición, 1980.

13. Germani, Gino. Política y Sociedad en una época en Transición: De la sociedad tradicional a la sociedad de masas. Editorial Paidós. Buenos Aires. 1964.

14. Bagú, Sergio. Estructura social de la colonia: Ensayo de historia comparada de América Latina. Edit. El Ateneo. Buenos Aires. 1952.

15. Freire, Gilberto. Casa Grande y Senzala: Formación de la familia brasileña bajo el régimen de economía patriarcal. Tomo I. Biblioteca de autores brasileños. Buenos Aires. 1942.

16. Ortiz, Fernando. Contrapunteo cubano del tabaco y del azúcar. Edit. Madrid: Cátedra, Edición de 2002. 1940.

17. Foster, George. Culture and Conquest: America's Spanish Heritage, Quadrangle Books, p. 3. 1960. 
18. Turner, F. Reassessing Political Culture, in Peter Smith (ed.) Latin America in Comparative Perspective, Westview Press, p.195. 1995.

19. Harrison, L. Underdevelopment Is a State of Mind, University Press of America, 1985, p. 165. 1985.

20. Harrison, L. Who Prosper? How Cultural Values Shape Economic and Political Success, Basic Books. p. 1 -3. 1992.

21. Harrison, L. The Pan-American Dream -- Do Latin America's Cultural Values Discourage True Partnership with the United States and Canada, Basic books, 1997, p. 4 - 27. 1997.

22. Wiarda, H. J. Introduction: Social Change, Political Development, and the Latin American Tradition, in Howard J. Wiarda, ed., Politics and Social Change in Latin America: Still a Distinct Tradition? Westview Press, p. 20. 1992.

23. North, D. Instituciones, Cambio Institucional y Desempeño Económico. Fondo de Cultura Económica. Economía Contemporánea. P. 43 - 75, 121- 136. 1990.

24. Vargas Llosa, Mario. América Latina y la Opción Liberal, in Barry Levine, ed., El Desafío Neoliberal: El fin del Tercermundismo en América Latina, Bogotá, 1992, pp.17-36.

\section{BIBLIOGRAFÍA DE CONSULTA}

Calles, J. Sobre algunos Mitos Fascistas: Un Texto Olvidado de Max Aub. Congreso Internacional del Centenario "Max Aub, testigo del siglo XX" Valencia.. En: http://www.uv.es/entresiglos/max/pdf/calles.pdf. 2003.

Fukuyama, Francis. Trust: the Social Virtues and the Creation of Prosperity. New York. Free Press. P. 6. 1995.

Germani, Gino. La sociología de la modernización. Editorial Paidós, Buenos Aires. 1969.

Lechner, Norbert (Compilador). Cultura política y democratización. Edición del Consejo Latinoamericano de Ciencias sociales FLACSO. Santiago de Chile. 1987. 
Romero, José Luis. Latinoamérica: las ciudades y las ideas. Colección Historia y Cultura. Edit. Siglo XXI editores Argentina. Tercera Edición (2001). 1976.

Simmel, Georg. Filosofía del dinero. Madrid, Instituto de Estudios Políticos, 1976, P. 12 y 13. 1977.

Veblen, Thorstein. The Instinct of Workmanship and the state of the Industrial Arts. New Brunswicks Reimp. 1990. P. 1 -37. 1914. 